\title{
Leaders
}

\section{Rugby football and anterior cruciate ligament injury}

In November 1997 the English National Rugby Union side played an arduous series of back to back matches against the three best sides in the world-namely, New Zealand, South Africa, and Australia. The tourists brought players with an enviable and potent mix of power and skill, as well as a small, but significant, number of anterior cruciate ligament (ACL) deficient knees that had not been reconstructed. The performance of these particular players challenges the current thinking that sportsmen and women with ACL ruptures must undergo reconstruction in order to compete at the highest level of a side-stepping sport such as rugby union, and this series was certainly played at the highest level! Our indications for surgery should be constantly appraised in the light of observing our patients.

An enormous volume of literature has been produced over the past 20 years on injuries to the ACL and their treatment. However, the number of well conducted scientific studies that can be relied upon to guide a clinician in the decision-making process is still small. At the very heart of this process is whether a player with a ruptured ACL should undergo reconstruction before returning to sport. Although evidence is accumulating that a reconstruction should be offered to high level athletes, there are still no absolute indications for surgery.

The loss of the ACL seems to affect both the mechanical and neuromuscular, or proprioceptive mechanisms that maintain the functional stability of the knee. The effect of this impairment on athletes can vary greatly. Some athletes will be asymptomatic at the highest level of activity, whereas others, at the other end of the spectrum, will be unable to walk normally without the knee repeatedly giving way. This diversity of outcomes after injury to the ACL must reflect a variation between individuals in the relative contribution of the ACL in generating the protective reflexes of the knee that operate to counter abnormal displacement of the tibia relative to the femur.

The mechanical role of the ACL in preventing anterior and anterolateral translation of the tibia relative to the femur has been extensively studied. Secondary restraints, such as the menisci, the collateral ligaments, and the shape of the articular surfaces themselves also play a part. In some individuals these secondary restraints can compensate sufficiently for the loss of the ACL with a negligible diminution of functional stability.

Perhaps of more interest, and certainly the subject of much of the current research, is the proprioceptive role of the ACL. Histological studies have now demonstrated proprioceptive nerve fibres in the intact ACL. ${ }^{1}$ The ability to sense an abnormal displacement of the knee, and to initiate protective muscle reflexes, is impaired in knees without an ACL. One such reflex is the contraction of the hamstring muscles to resist anterior tibial translation in response to an anteriorly directed load applied to the back of the tibia. Beard et al from this centre showed that the delay between application of the load and contraction of the hamstrings was significantly greater in patients' ACL deficient knees than in their normal knees. ${ }^{2}$ They proposed that the ACL sensed abnormal displacement of the tibia and through a spinal reflex effected a contraction in the hamstring muscles to resist the displacement. They then showed that reconstruction of the ACL or a specific hamstring predominant rehabilitation programme could improve the delay in reflex hamstring contraction.

My own observation of the rugby players who are continuing to play on unreconstructed ACL deficient knees is that they are all very muscular, squat players with a high power to weight ratio. Their relatively short lever arms and powerful muscles would favour any muscle driven protective reflex and might be an important part of the jigsaw that makes up the picture of a functionally stable knee. To date, the morphology of athletes with ACL deficiency has not received much attention but may be an important factor in predicting the outcome. A prospective clinical trial to evaluate this may not be possible so reliance on case-control studies will be needed.

The aim when treating athletes with ACL deficiency should be to get them back to their previous level of activity while protecting their knees from further injury, particularly meniscal injury due to subtle instability. At present it is impossible to predict with complete accuracy who will require reconstructive surgery but Daniel and Fithian suggest that the number of hours/year participation in level I/II sport combined with the degree of knee laxity can be helpful. ${ }^{3}$ Patients in a high risk group who did not undergo reconstruction were estimated to have a $40 \%$ chance of requiring late meniscal or ACL surgery within five years of the original injury. If reconstruction is not advocated you need to be clear that the athlete is not a "knee abuser", as Frank Noyes termed them, who simply denies episodes of instability but continues to do further damage to his joint every Saturday afternoon. I would suggest that rugby players might fall into this last category more often than not!

Nuffield Orthopaedic Centre

JONATHAN WEBB

Windmill Road

Headington

Oxford OX3 $7 L D$

1 Biedert RM, Stauffer E, Friederich NF. Occurrence of free nerve endings in the soft tissue of the knee joint. A histologic investigation. Am f Sports Med 1992;20:430-3.

2 Beard D, Kyberd P, Ferguson C, et al. Proprioception after rupture of the anterior cruciate ligament. F Bone foint Surg Br 1993;75:311-15.

3 Daniel D, Fithian D. Indications for ACL surgery. Arthroscopy 1994;10:43141. 